\title{
ASYMPTOTIC ESTIMATES FOR SYMMETRIC VORTEX STREETS
}

\author{
G. KEADY ${ }^{1}$
}

(Received 9 May 1983; revised 13 February 1984)

\begin{abstract}
Steady plane inviscid symmetric vortex streets are flows defined in the strip $R \times(0, b)$ and periodic in $x$ with period $2 a$ in which the flow in $(-a, a) \times(0, b)$ is irrotational outside a vortex core on which the vorticity takes a prescribed constant value. A family of such vortex street flows, characterised by a variational principle in which the area $\left|A_{\alpha}\right|$ and the centroid $y_{c}$ of the vortex core $A_{\alpha}$ are fixed, will be considered. For such a family, indexed by a parameter $\alpha$, suppose that the cores $A_{\alpha}$ become small in the sense that
\end{abstract}

$$
\left(\operatorname{area}\left(A_{\alpha}\right)\right) / y_{c}^{2} \rightarrow 0 .
$$

Asymptotic estimates on functionals such as flux constant and speed are obtained.

\section{Introduction}

Throughout this paper, $\Omega$ is the rectangle $(-a, a) \times(0, b)$. For all theorems proved in this paper $a$ and $b$ are bounded, though in discussion, in particular comparison with other published results, one or both of $a$ or $b$ may be infinite.

When $a$ is bounded the situation is that of a periodic vortex street. See Figure 2c. The case that both $a$ and $b$ are infinite is that of vortex pairs. See Figure 1 .

Vortex pairs travel downwards behind the wings of aircraft in steady horizontal flight. At a large distance behind the aircraft the flow is approximately two-dimensional. With respect to axes such that the vortex pair is stationary the streamline pattern is as in Figure 1. Just one half of the vortex pair is shown. The other half of the flow is the mirror image in the line $y=0$.

\footnotetext{
${ }^{1}$ Mathematics Department, University of Western Australia, Nedlands, W.A. 6009. c Copyright Australian Mathematical Society 1985, Serial-fee code 0334-2700/85
} 


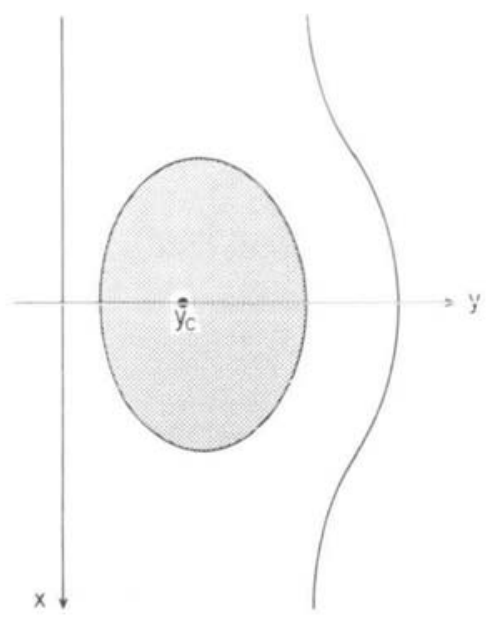

Figure 1 . The flow pattern of vortex pairs. The shaded region is the vortex core.

A nineteenth century model of such flows was to suppose that the vorticity in each of the half-planes $y>0$ and $y<0$ is concentrated at the points $\left(0, y_{c}\right)$ and $\left(0,-y_{c}\right)$ respectively. If the circulation about the vortex at $\left(0, y_{c}\right)$ is $\lambda \alpha$, Lamb [11] gives the speed $V_{\text {sing }}$ of the fluid at infinity

$$
V_{\text {sing }}=\lambda \alpha /\left(4 \pi y_{c}\right) \text {. }
$$

The more realistic model of vortex pairs considered in this paper supposes that the vorticity is constant $\lambda$ in the vortex core $A_{\alpha} \subset\{(x, y) \mid y>0\}$. The area of $A_{\alpha}$ is $\alpha$; the centroid of $A_{\alpha}$ is $\left(0, y_{c}\right)$.

The partial differential equation problem describing the vortex street, and the special case of vortex pairs, is problem $(\mathrm{P})$ defined in Section 2.

A functional of the flow is the flux $k$ of fluid between the axis $y=0$ and the boundary of the vortex core $A$. A second functional of the flow is $V$, which corresponds to the speed of the vortex street or vortex pair.

Lichtenstein $[12,13]$ used contraction mapping techniques to establish the existence of such solutions when $\alpha / y_{c}^{2}$ is sufficiently small, which have cores $A_{\alpha}$ close to circular. Outside the core the solutions have the stream function $\psi$ behaving like a point vortex

$$
\psi(x, y)-\lambda \alpha G\left(x, y, 0, y_{c}\right),
$$

where $G$ denotes the Green function for $-\Delta$ on $\Omega$ with the boundary conditions described below (in Section 2) in the formulation of problem (P). For these solutions

$$
k \lambda \alpha-\frac{(\lambda \alpha)^{2}}{4 \pi} \log \frac{4 \pi y_{c}^{2}}{\alpha}
$$


and

$$
\begin{aligned}
& V \lambda \alpha-(\lambda \alpha)^{2} /\left(4 a \tanh \frac{\pi y_{c}}{a}\right) \quad \text { in the case } b=\infty, \\
& V \lambda \alpha-(\lambda \alpha)^{2} /\left(4 b \tan \frac{\pi y_{c}}{b}\right) \text { in the case } a=\infty .
\end{aligned}
$$

(as in Lamb [11], Rosenhead [19]). Except for the local result associated with the contraction mapping proof this author is unaware of uniqueness results.

Modern global existence results are now available. Some of these are based on variational principles. Benjamin [1] argued on physical grounds that problem (V), defined in Section 2, is particularly appropriate.

It seems a reasonable conjecture that the 'classical' asymptotically circular solutions described are the solutions of the variational problem (V), when the given parameters $\alpha$ and $y_{c}$ are such that $\alpha / y_{c}^{2}$ is sufficiently small. Proving results towards establishing this conjecture is one of the motivations for this paper.

The phrase 'asymptotically tight' will now be defined. It will be used to describe inequalities on some functional $F(\alpha)$ of the solutions, $\alpha$ being the area of $A_{\alpha}$. Let $F_{\text {circ }}(\alpha)$ denote the value of the functional $F$ when evaluated for small $\alpha$ on the classical nearly-circular solutions of Lichtenstein $[12,13]$ described above. The estimate

$$
F(\alpha) \leqslant F_{\text {bound }}(\alpha),
$$

is said to be asymptotically tight if

$$
\frac{F_{\text {bound }}(\alpha)}{F_{\text {circ }}(\alpha)} \rightarrow 1 \quad \text { as } \alpha=\operatorname{area}\left(A_{\alpha}\right) \rightarrow 0 .
$$

Asymptotic estimates for vortex rings are published in Friedman and Turkington [6]. Asymptotic estimates for vortex pairs and some related plane flows are proved in Turkington $[22,23]$. The proofs in our paper are different. There are several reasons for this, one of which is that we use additional isoperimetric inequalities.

The plan of the paper is now outlined.

The mathematical problem is formulated in Section 2. The entire strategy is exemplified in the special case of vortex pairs in Section 3. Some hydrodynamical topics related to the general problem are discussed in Section 4, though the results there are not necessary for the remainder of the paper.

Sections 5 and 6 are devoted to essential preliminary results. (Section 7 is devoted to capacity questions, and is not needed for Theorem 8.1.)

The two obvious functionals to estimate are the flux constant $k$ and the speed $V$. The first asymptotic estimate, Theorem 8.1 , concerns the quantity

$$
\min \{k, k+V b\} \text {. }
$$


In Section 7 of [10] it is shown that $y_{c}<b / 2$ implies $V>0$, and hence that the estimate of Theorem 8.1 gives the flux constant.

There are various ways in which the asymptotic estimates of Theorem $8.1 \mathrm{can}$ be used. Berger and Fraenkel [2] suggested that the electrostatic capacity of $A$, $\operatorname{cap}(A)$, is a useful functional for obtaining information on the geometry of $A$. (See also [3].) The development of their ideas for this problem is outlined in this paper with full proofs given in [10]. Thus Section 7 is concerned with capacity questions. The results of Sections 7 and 8 together ensure that for the variational solutions with connected cores

$$
\frac{\operatorname{diam}\left(A_{\alpha}\right)}{y_{c}} \rightarrow 0 \quad \text { as } \frac{\operatorname{area}\left(A_{\alpha}\right)}{y_{c}^{2}} \rightarrow 0 .
$$

This result is comparable with those, for different problems, of Berger and Fraenkel [2] and of the Appendices in Keady and Norbury [8] and in Keady [9].

In Section 7 of [10] the asymptotic estimates are improved upon, especially in connection with the speed $V$.

\section{The mathematical problems}

The (set-valued) function $H$ is defined by

$$
H(t)=\{0\} \text { for } t<0, H(t)=\{1\} \text { for } t>0 \text {, and } H(0)=[0,1] .
$$

The symmetric vortex street problem, problem (P), is to find, given $\lambda>0$, the functions $\psi$ and associated numbers $k$ and $V$, that is the triple $(\psi, k, V)$, solving

$$
\left.\begin{array}{r}
-\Delta \psi\left(=-\psi_{x x}-\psi_{y y}\right) \in \lambda H(\psi-q) \text { in } \Omega, \\
\psi(x, 0)=\psi(x, b)=0,-a \leqslant x \leqslant a, \\
\psi_{x}( \pm a, y)=\psi_{x}(0, y)=0,0 \leqslant y \leqslant b,
\end{array}\right\}
$$

with $q=k+V y$.

Define the vortex core

$$
A_{\psi}=\{(x, y) \in \Omega \mid \psi(x, y)>q(x, y)\},
$$

and omit the subscript $\psi$ when no confusion could arise, and allow other subscripts when this helps.

This paper is concerned solely with solutions for which the core boundary,

$$
\partial A_{\psi}=\{(x, y) \in \bar{\Omega} \mid \psi(x, y)=q(x, y)\},
$$

is of zero measure. (See Appendix B of [10] for the proof that this is so for variational solutions.) 
An alternative useful way to consider the problem $(\mathrm{P})$ is in terms of the triple $(A, k, V)$. Here $A$ is an open subset of $\Omega$ and $k$ and $V$ are numbers such that when $(\psi, k, V)$ is defined as follows it solves the preceding elliptic partial differential equation and boundary conditions, problem (P). Given $A, \psi \in$ $W_{2}^{2}(\Omega) \cap C^{1, \nu}(\bar{\Omega})$ for any $\nu$ satisfying $0<\nu<1$, is defined to satisfy

$$
-\Delta \psi= \begin{cases}\lambda & \text { in } A, \\ 0 & \text { in } \Omega \backslash \bar{A},\end{cases}
$$

and the boundary conditions on $\psi$ stated above. The preceding elliptic partial differential equation boundary-value problem $(\mathrm{P})$ is solved in the sense that

$$
\psi(x, y)>q=k+V y \Leftrightarrow(x, y) \in A .
$$

We shall refer to the triple $(A, k, V)$ as a solution of problem $(\mathrm{P})$.

The solutions of problem (P) which are independent of $x$ will be called (unidirectional) streams. Explicit expressions for these solutions are given in Berger and Fraenkel [2] and in Section 4 here and, in more detail, in Section 2.1 of [10].

Define areas and centroids by

$$
|A|=\operatorname{area}(A), \quad y_{c}=\frac{1}{|A|} \int_{A} y .
$$

Throughout this paper $\lambda$ is taken to be a fixed positive number and $y_{c}$ a fixed number satisfying $0<y_{c} \leqslant b / 2$. Let $\alpha_{0}$ be the area of the largest disc, centre $\left(0, y_{c}\right)$, contained in $\Omega$. Also $\alpha=\operatorname{area}\left(A_{\alpha}\right)$ is assumed to satisfy $\alpha<\alpha_{0}$.

The following variational problem has been considered (in [1], [14], [22], [23]) in connection with problem (P). Define, for $\alpha<\alpha_{0}$ :

$$
\begin{gathered}
\mathscr{S}_{\alpha}=\left\{D \subset \Omega \mid D \text { is open, }|D|=|\bar{D}|=\alpha, \text { centroid }(D)=\left(0, y_{c}\right)\right\} ; \\
E(D)=\frac{\lambda^{2}}{2} \int_{D \times D} G \text { for } D \in \mathscr{S}_{\alpha},
\end{gathered}
$$

where $G$ is the Green function for $-\Delta$ on $\Omega$ with the given boundary conditions. A solution of problem $\left(\mathrm{V}\left(\mathscr{S}_{\alpha}\right)\right)$ is an $A$ belonging to $\mathscr{S}_{\alpha}$ satisfying

$$
E(A)=\max _{D \in \mathscr{S}_{\alpha}} E(D) .
$$

The variational problem will be considered allowing the competitors to be members of various subsets $\mathscr{C}_{\alpha}$ of $\mathscr{S}_{\alpha}$. Define, for $\alpha<\alpha_{0}$,

$$
B_{\alpha}=\left\{(x, y) \mid x^{2}+\left(y-y_{c}\right)^{2}<\alpha / \pi\right\} .
$$

The subclasses $\mathscr{C}_{\alpha}$ must have the property that $B_{\alpha}$ belongs to $\mathscr{C}_{\alpha}$.

Problem (V( $\left.\mathscr{C}_{\alpha}\right)$ ), abbreviated to problem (V) below, is to find $A$ belonging to $\mathscr{C}_{\alpha}$ such that

$$
E(A)=\max _{D \in \mathscr{C}_{a}} E(D)
$$


The set $\mathscr{C}_{\alpha}$ is to be taken such that the solutions to problem (V) exist, solve problem (P), have $A$ symmetrised with respect to $x$, have the closure of $A$ not touching $y=0$ or $y=b$, and have the boundary of $A$ smooth enough for applications of the divergence theorem.

In an Appendix of [10] existence results, for (solutions not independent of $x$ for) problem (V), are described. Existence for two separate special cases was previously available, or readily deduced using the same methods, in Turkingion $[22,23]$. These two special cases are (i) the base $b$ infinite, in particular the vortex pair problem when both $a$ and $b$ are infinite, and (ii) (see also [14]) the symmetric linear array problem, $y_{c}=b / 2$ with the flow symmetric about $y=b / 2$. In case (ii) the connectedness of the cores (useful in Sections 7.2 and 8.1 below) has also been proved. (The notation of this paper differs slightly from that of [10] in that, in [10], the classes $\mathscr{C}_{\alpha}$ also had $A$ connected.)

\section{Vortex pairs}

There are various special cases where certain aspects simplify. It may assist in following the general strategy of the paper to see this strategy illustrated in a special case. For this reason, and to allow direct comparison with Turkington [23], the case when both $a$ and $b$ are infinite is now considered. The treatment is formal in order to avoid digressions, concerning behaviour at infinity, which are not needed elsewhere in this paper.

(The vortex pairs mentioned in the title of this section are obtained by adding the reflection across the line $y=0$.)

For the material in Sections 6 to 8, anticipated in this section, define for solutions $A, k, V$ of problem (P),

$$
\Psi=\psi-k-V y .
$$

The methods of Section 5 yield, for vortex pairs, the following inequality satisfied by solutions of problem (V),

$$
2 E\left(A_{\alpha}\right) \geqslant \frac{(\lambda \alpha)^{2}}{8 \pi}\left\{1+2 \log \frac{4 \pi y_{c}^{2}}{\alpha}\right\} \quad(a=b=\infty) .
$$

The methods of Section 6 yield, for vortex pairs, the following for solutions of problem (P),

$$
\lambda \alpha k=2 E\left(A_{\alpha}\right)-3 \lambda \int_{A_{\alpha}} \Psi \quad(a=b=\infty)
$$




$$
\lambda \int_{A_{a}} \Psi \leqslant \frac{1}{8 \pi}(\lambda \alpha)^{2} .
$$

(When the results of the preceding two sentences are combined they lead to a lower bound for $k$.)

The methods of Section 7.1 yield, for vortex pairs, the following inequality for solutions of problem (P),

$$
k \operatorname{cap}\left(A_{\alpha}\right) \leqslant \lambda \alpha .
$$

When the results of the preceding three paragraphs are combined it is found that

$$
\operatorname{cap}\left(A_{\alpha}\right) \leqslant \frac{4 \pi}{\log \left(4 \pi y_{c}^{2} / \alpha\right)-1},
$$

so that $\operatorname{cap}\left(A_{\alpha}\right) \rightarrow 0$ as $\alpha \rightarrow 0$.

(It is possible to parallel the above development rather closely when $a$ is infinite but $b$ is finite. Alternatively there are considerable simplifications to the arguments of Sections 5 to 8 if, instead of taking zero Neumann data as in problem (P), the case of zero Dirichlet data at $x=-a$ and at $x=+a$ is considered instead. The details can be provided by straightforward extensions of the methods of this paper. One reason why the development in Sections 5 to 8 is longer than that of this section is that the formula for $k$ generalising that above involve boundary integral terms which must be estimated. The Neumann boundary conditions further complicate these estimates.)

\section{Hydrodynamic topics}

\subsection{Unidirectional streams}

Because of the Neumann boundary conditions at $x=-a$ and at $x=+a$ there are solutions of problem $(\mathrm{P})$ with

$$
A=A\left[y_{-}, y_{+}\right]=(-a, a) \times\left(y_{-}, y_{+}\right) .
$$

Such solutions also solve the variational problems when these are restricted to one dimension. These solutions will be called (unidirectional) streams. For further details see Section 2.1 of [10].

Looking ahead to Theorem 5.2 it will be seen that, for $\operatorname{area}(A)$ sufficiently small, if there is a solution to problem (V), it must depend upon $x$.

\subsection{Other solutions}

Besides the unidirectional streams discussed in Section 4.1 we expect that there are solutions to problems (P) and (V) of the character illustrated in Figure 1. Numerical evidence for this is given in case $y_{c}=b / 2, V=0$ in Saffman and Szeto [21], Pierrehumbert and Widnall [17]. 


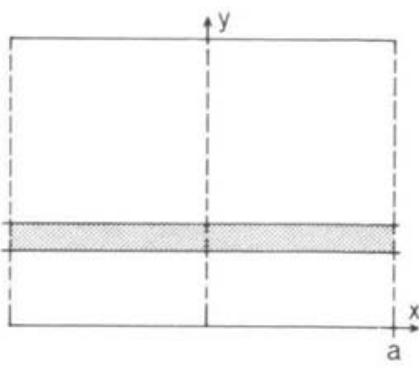

(a)

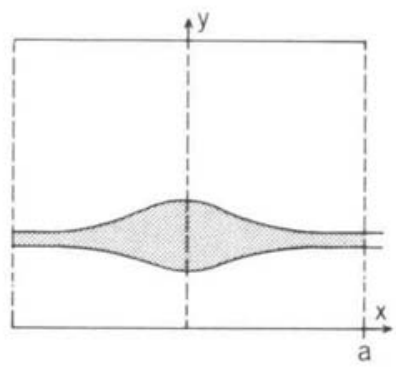

(b) 'waves'

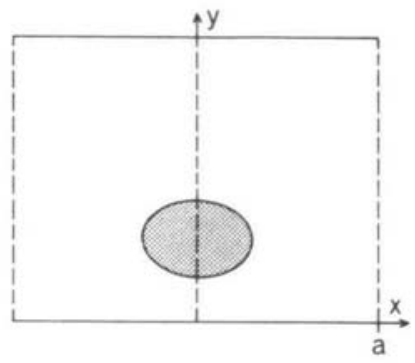

(c) 'patches'

Figure 2.

This paper is concerned with patches of vorticity as illustrated in Figure 2(c).

The study of the extrema of the energy $E$, and so in particular the solutions of problem (V), is motivated by certain stability considerations. For the symmetric vortex street we expect the solutions, like the singular street, to be unstable to disturbances of twice the period of the street and to be saddle points of the energy in this larger setting. The discussion in Benjamin [1] suggests in an intuitive rather than rigorous way, the connection between any existence results for problem (V) and results concerned with stability with respect to disturbances to the same period as the street.

\section{Potential theoretic preliminaries}

Given a set $D \in \mathscr{S}_{\alpha}$, define

$$
\psi_{D}=\lambda \int_{D} G
$$

Then we have the following characterisations of $E(D)$. 
LEMMA 5.1.

$$
\lambda^{2} \int_{D \times D} G=\lambda \int_{D} \psi_{D}=\int_{\Omega}\left|\nabla \psi_{D}\right|^{2}
$$

Proof. The first equality is immediate from the definition of $\psi_{D}$. The second equality follows on using the divergence expression

$$
\int_{\Omega} \operatorname{div}\left(\psi_{D} \nabla \psi_{D}\right)=0
$$

We follow Turkington [22] in defining the function

$$
H\left(x, y, 0, y_{c}\right)=-G\left(x, y, 0, y_{c}\right)-\frac{1}{4 \pi} \log \left(x^{2}+\left(y-y_{c}\right)^{2}\right),
$$

to define a harmonic function in $\Omega$ continuous on the closure of $\Omega$.

TheOREM 5.2. Let $B=B\left(\left(0, y_{c}\right), p\right) \subset \Omega$. Then

$$
\psi_{B}=\lambda \int_{B} G
$$

may be written as follows:

$$
\begin{aligned}
& \psi_{B}(x, y)= \frac{\lambda}{4}\left(\rho^{2}-x^{2}-\left(y-y_{c}\right)^{2}\right)-\lambda \pi \rho^{2} H\left(x, y, 0, y_{c}\right) \\
&-\frac{\lambda \rho^{2}}{4} \log \rho^{2} \text { in } B, \\
& \psi_{B}(x, y)=\lambda \pi \rho^{2} G\left(x, y, 0, y_{c}\right) \text { in } \Omega \backslash B .
\end{aligned}
$$

Define

$$
q_{b}=\lambda|B|\left(\frac{1}{4 \pi} \log \frac{\pi}{|B|}-H\left(0, y_{c}, 0, y_{c}\right)\right)
$$

and observe that

$$
q_{b} \sim\left(\frac{\lambda|B|}{4 \pi} \log \frac{4 \pi y_{c}^{2}}{|B|}\right)(1+o(1)) \quad \text { as } \frac{|B|}{y_{c}^{2}} \rightarrow 0 .
$$

Then

$$
2 E(B)=\frac{\lambda^{2}|B|^{2}}{8 \pi}+\lambda|B| q_{b}
$$

REMARK. The approach for the calculation of $E(D)$ may be useful for other domains $D$ (such as ellipses) for which both the torsion function and $\psi_{D}$ are 
known explicitly. (For elliptical $D$ see Moore and Saffman [15], Saffman [20].) This approach is now presented in its general form.

Recall that

$$
\psi_{D}=\lambda \int_{D} G
$$

The torsion problem for $D$ is, and the function $\Psi_{D}$ its solution satisfy,

$$
-\Delta \Psi_{D}=\lambda \text { in } D, \quad \Psi_{D}=0 \text { on } \partial D .
$$

It follows, omitting the subscripts $D$,

$$
2 E(D)=\lambda \int_{D} \psi=\lambda \int_{D} \Psi+\lambda \int_{D}(\psi-\Psi)
$$

The divergence theorem gives another representation of the latter term:

$$
\lambda \int_{D}(\psi-\Psi)=-\int_{D} \operatorname{div}((\psi-\Psi) \nabla \Psi-\Psi \nabla(\psi-\Psi))=-\int_{\partial D}(\psi-\Psi) \frac{\partial \Psi}{\partial n},
$$

where $n$ is the outward normal from $D$.

Proof of TheOREM 5.2. It is straightforward to check the representation (5.2) of $\psi_{B}$. Consider the above representation (5.5) when $D=B$, the disc in the statement of the theorem. The mean-value theorem for harmonic functions gives

$$
2 E(B)=\lambda \int_{B} \Psi_{B}+\lambda|B|\left(\psi_{B}\left(0, y_{c}\right)-\Psi_{B}\left(0, y_{c}\right)\right),
$$

which is precisely the expression in equation (5.4).

\section{Some identities}

\subsection{The torsion problem}

LEMMa 6.1 (ST. VeNANT INEQUALITY). For solutions $\Psi$ of the torsion problem

$$
\begin{aligned}
-\Delta \Psi=\lambda & \text { in } D, \\
\Psi=0 & \text { on } \partial D,
\end{aligned}
$$

the following inequality holds:

$$
\lambda \int_{D} \Psi \leqslant \frac{1}{8 \pi}(\lambda|D|)^{2} .
$$

For proof see Polya and Szego [18] or Payne [16].

The St. Venant inequality for domain $A$ will be used when $A$ is (the core of) a solution of problem (P), and in Section 8 the closure of $A$ will be required to be 
properly contained in $\Omega$, in order that the inequality will apply. For the case that $A$ might have several components inequality (6.1) is established by combining the St. Venant inequalities for each component.

6.2. Identities for problem (P).

For solutions $A, k, V$ of problem (P) define

$$
\Psi=\psi-k-V y \text {. }
$$

LEMMA 6.2. For solutions of problem (P), writing $q_{c}=k+V y_{c}$, the following identity holds:

$$
2 E(A)=\lambda|A| q_{c}+\lambda \int_{A} \Psi
$$

Proof. Immediate from definitions and Lemma 5.1.

LEMMA 6.3. For solutions of problem $(\mathrm{P})$,

$$
\begin{gathered}
\lambda|A| V y_{c}=\int_{A} \lambda y_{c} \psi_{y}=\int_{A} \lambda y \psi_{y}+\lambda \int_{A} \Psi, \\
-\lambda \int_{A}\left(y-y_{c}\right) \psi_{y}=\lambda \int_{A} \Psi,
\end{gathered}
$$

and, provided the closure of $A$ is a proper subset of $\Omega$,

$$
-\lambda \int_{A} x \psi_{x}=\lambda \int_{A} \Psi
$$

so that

$$
-\lambda \int_{A}\left(x \psi_{x}+\left(y-y_{c}\right) \psi_{y}\right)=2 \lambda \int_{A} \Psi
$$

Proof. Equation (6.3) is possibly the most important for subsequent applications. It can be proved directly as follows:

$$
\begin{aligned}
-\lambda \int_{A}\left(x \psi_{x}+\left(y-y_{c}\right) \psi_{y}\right) & =-\lambda \int_{A}\left(x \Psi_{x}+\left(y-y_{c}\right) \Psi_{y}\right) \\
& =-\lambda \int_{A} \operatorname{div}\left(\left(x, y-y_{c}\right) \Psi\right)+2 \lambda \int_{A} \Psi \\
& =2 \lambda \int_{A} \Psi .
\end{aligned}
$$

The first (and second) lines follow from

$$
\left(y-y_{c}\right) \psi_{y}+q+\Psi=\left(\left(y-y_{c}\right)(q+\Psi)\right)_{y},
$$


and integrating over $A$

$$
\int_{A}\left(y-y_{c}\right) \psi_{y}+\left(k+V y_{c}\right)|A|+\int_{A} \Psi=\int_{A}\left(\left(y-y_{c}\right) q\right)_{y}=\left(k+V y_{c}\right)|A| .
$$

Below, and in Section 8 the following notation will be used, mostly within integrations. Where no confusion can arise write $f(0)=f(x, 0), f(b)=f(x, b)$ and $f(a)=f(a, y)$.

COROLLARY 6.3. With the hypotheses of Lemma 6.3:

$$
\begin{gathered}
-\lambda \int_{A} \Psi+\lambda|A| V y_{c}=\int_{A} y \psi_{y}=\frac{1}{2} \int_{\Omega}\left(\psi_{y}^{2}-\psi_{x}^{2}\right)-\frac{b}{2} \int_{-a}^{a} \psi_{y}(b)^{2}, \\
-\lambda \int_{A} \Psi=\lambda \int_{A} \psi_{x}=-\frac{1}{2} \int_{\Omega}\left(\psi_{y}^{2}-\psi_{x}^{2}\right)+a \int_{0}^{b} \psi_{y}(a)^{2}, \\
\lambda|A| V=\lambda \int_{A} \psi_{y}=\frac{1}{2} \int_{-a}^{a} \psi_{y}(0)^{2}-\frac{1}{2} \int_{-a}^{a} \psi_{y}(b)^{2} .
\end{gathered}
$$

Proof. The first two identities, (6.4) and (6.5), follow from

$$
\frac{b}{2} \int_{-a}^{a} \psi_{y}(b)^{2}=\frac{1}{2} \int_{\Omega}\left(y\left(\psi_{y}^{2}-\psi_{x}^{2}\right)\right)_{y}, \quad a \int_{0}^{b} \psi_{y}(a)^{2}=\frac{1}{2} \int_{\Omega}\left(x\left(\psi_{y}^{2}-\psi_{x}^{2}\right)\right)_{x} .
$$

Equation (6.6) follows from

$$
\frac{1}{2} \int_{-a}^{a}\left(\psi_{y}(b)^{2}-\psi_{y}(0)^{2}\right)=\frac{1}{2} \int_{\Omega}\left(\left(\psi_{y}^{2}-\psi_{x}^{2}\right)_{y}+\left(2 \psi_{y} \psi_{x}\right)_{x}\right)
$$

\section{Some results concerning capacity}

\subsection{Electrostatic capacity}

As stated in the Introduction a major result of Sections 7 and 8 will be to demonstrate that, for connected variational solutions,

$$
(\operatorname{diam}(A)) / y_{c} \rightarrow 0 \quad \text { as }(\operatorname{area}(A)) / y_{c}^{2} \rightarrow 0 .
$$

In Section 8.1 it will be shown that, for variational solutions,

$$
\operatorname{cap}(A) \rightarrow 0 \text { as }(\operatorname{area}(A)) / y_{c}^{2} \rightarrow 0 .
$$

This, with the result of Section 7.2, yields the diameter result. (As explained in Turkington [22, 23], this strategy, which is due to Berger and Fraenkel [2], is not the only one.) 
The definition of $\operatorname{cap}(A)=\operatorname{cap}(A, \Omega)$, which differs from the usual because of the Neumann boundary conditions at $x=+a$ and at $x=-a$, is now given. Define, for closed sets $F$, $\operatorname{cap}(F)=\inf \left\{\int_{\Omega}|\nabla u|^{2} u \in W_{2}^{1}, u \geqslant 1\right.$ on $F$

$$
\left.u=0 \text { on } y=0, b, u_{x}=0 \text { on } x=0, \pm a\right\} \text {, }
$$

and for open sets $D, \operatorname{cap}(D)=\operatorname{cap}(\bar{D})$. It is easy to verify the usual properties such as domain monotonicity

$$
\operatorname{cap}\left(F^{\prime}\right) \leqslant \operatorname{cap}(F) \text { if } F^{\prime} \subseteq F .
$$

LemMa 7.1. Let $A, k, V$ solve problem $(\mathrm{P})$ with vortex core $A$, such that the closure of $A$ is contained in $\Omega$. Let

$$
\begin{gathered}
q=k+V y, \\
q_{\min }=\min _{\partial A} q, \quad q_{\max }=\max _{\partial A} q .
\end{gathered}
$$

Then

$$
q_{\min } \operatorname{cap}(A) \leqslant \lambda|A| \leqslant q_{\max } \operatorname{cap}(A) .
$$

Proof. See [10].

\subsection{The capacity of connected sets}

As in Fraenkel [4], Steiner symmetrisation techniques are used in [10] to establish that, for connected open sets $D_{\alpha}$

$$
\operatorname{cap}\left(D_{\alpha}, \Omega\right) \rightarrow 0 \Rightarrow \operatorname{diam}\left(D_{\alpha}\right) \rightarrow 0 .
$$

See also [5] for definitions of Steiner symmetrisation.

\section{Asymptotic estimates}

Henceforth assume that the closure of $A$ is a proper subset of $\Omega$. In the notation of Section 7.1,

$$
q_{\min } \geqslant \min \{k, k+V b\}
$$

and

$$
\operatorname{cap}(A) \leqslant \lambda|A| / q_{\min } .
$$

It will be shown that, for $\alpha=\operatorname{area}(A)$ sufficiently small

$$
\min \{k, k+V b\} \geqslant k_{b} \text {, }
$$

for suitable $k_{b}$. 
LEMMA 8.1. For solutions of problem $(\mathrm{P})$

$$
k \lambda|A|=2 E(A)-\lambda \int_{A} \Psi-\frac{1}{2} \int_{\Omega}\left(\psi_{y}^{2}-\psi_{x}^{2}\right)+\frac{b}{2} \int_{-a}^{a} \psi_{y}(b)^{2},
$$

and

$$
(k+V b) \lambda|A|=2 E(A)-\lambda \int_{A} \Psi-\frac{1}{2} \int_{\Omega}\left(\psi_{y}^{2}-\psi_{x}^{2}\right)+\frac{b}{2} \int_{-a}^{a} \psi_{y}(0)^{2},
$$

so that

$$
\lambda|A| \min \{k, k+V b\} \geqslant E(A)-2 \lambda \int_{A} \Psi .
$$

For solutions of problem $(\mathrm{V})$ also solving problem $(\mathrm{P})$

$$
\min \{k, k+V b\} \geqslant k_{b} \quad \text { with } k_{b}=\frac{E(B)}{\lambda|A|}-\frac{\lambda|A|}{4 \pi} .
$$

where $E(B)$ is defined in Theorem 5.2.

Proof. Identity (8.1a) follows from identities (6.2a) and (6.4), and then identity (8.1b) follows from identities (8.1a) and (6.6). The manipulations to arrive at inequality (8.2) are done separately for $k$ and then for $(k+V b)$. They involve only trivial inequalities, replacing the boundary integral term by zero and

$$
\int_{\Omega}\left(\psi_{y}^{2}-\psi_{x}^{2}\right)-b \int_{-a}^{a} \psi_{y}(b)^{2} \leqslant \int_{\Omega}\left(\psi_{y}^{2}-\psi_{x}^{2}\right) \leqslant 2 E(A) .
$$

Inequality (8.3) then follows from the St. Venant inequality (6.1) and the fact that $E(A) \geqslant E(B)$.

Inequality (8.3) is not asymptotically tight for small nearly-circular cross-sectional cores. See relation (1.2).

There are two special cases when inequality (8.4) (and then inequality (8.3)) can be improved. There are (i) the case $a$ infinite, and (ii) the case $y=b / 2, V=0$.

(i) In the case $a$ infinite, by identity (6.5),

$$
\int_{\Omega}\left(\psi_{y}^{2}-\psi_{x}^{2}\right)=2 \lambda \int_{A} \Psi
$$

and then the St. Venant inequality (6.1) improves upon (8.4)

(ii) In the case $y_{c}=b / 2, V=0$, by identity (6.4)

$$
\int_{\Omega}\left(\psi_{y}^{2}-\psi_{x}^{2}\right)-b \int_{-a}^{a} \psi_{y}(b)^{2}=-\lambda \int_{A} \Psi \leqslant 0
$$

and this improves upon (8.4).

The discussion at the beginning of this section establishes:

THEOREM 8.2. For a family of solutions $A_{\alpha}$ of problem (V) also solving problem (P)

$$
\operatorname{cap}\left(A_{\alpha}\right) \rightarrow 0 \text { as } \alpha=\operatorname{area}\left(A_{\alpha}\right) \rightarrow 0 .
$$




\subsection{The diameter result for connected cores}

Using the results of Section 7.2 with Theorem 8.2 the following is established:

COROLlaRY 8.2. For a family of (connected) solutions $A_{\alpha}$ of problem (V) also solving problem $(\mathrm{P})$

$$
\operatorname{diam}\left(A_{\alpha}\right) / y_{c} \rightarrow 0 \text { as } \operatorname{area}\left(A_{\alpha}\right) / y_{c}^{2} \rightarrow 0 .
$$

Further implications of this, developing the methods of [2] for this problem, are given in [10]. The results there establish that, for variational solutions with connected cores, the asymptotic relation (1.1) is satisfied.

\section{References}

[1] T. B. Benjamin, “The alliance of practical and analytical insights into the nonlinear problems of fluid mechanics", in Applications of methods of functional analysis to problems in mechanics, Symposium IUTAM/IMU Marseilles, Sept. 1975 (eds. P. Germain and B. Nayroles), Lecture Notes in Math. 503 (Springer, Berlin, 1976), 8-29.

[2] M. S. Berger and L. E. Fraenkel, "Nonlinear desingularization in certain free-boundary problems”, Comm. Math. Phys. 77 (1980), 149-172.

[3] L. A. Caffarelli and A. Friedman, "Asymptotic estimates for the plasma problem ", Duke Math. J. 47(1980), 705-762.

[4] L. E. Fraenkel, "A lower bound for electrostatic capacity in the plane", Proc. Roy. Soc. Edinburgh Sect. A 88 (1981), 267-273.

[5] L. E. Fraenkel and M. S. Berger, "A global theory of steady vortex rings in an ideal fluid", Acta Math. 132(1974), 13-51.

[6] A. Friedman and B. Turkington, "Vortex rings: existence and asymptotic estimates", Trans. Amer. Math. Soc. 268 (1981), 1-38.

[7] B. Gidas, W. Ni and L. Nirenberg, "Symmetry via the maximum principle," Comm. Math. Phys. 68(1979), 209-243.

[8] G. Keady and J. Norbury, "A semilinear elliptic eigenvalue problem, part II”, Proc. Roy. Soc. Edinburgh Sect. A 87 (1980), 83-109.

[9] G. Keady, “An elliptic boundary-value problem with a discontinuous nonlinearity", Proc. Roy. Soc. Edinburgh Sect. A 91 (1981), 161-174.

[10] G. Keady, "Existence and asymptotic estimates for symmetric vortex streets", Res. Rep. Math. Dep. Univ. Western Australia (January 1983).

[11] H. Lamb, Hydrodynamics (Cambridge University Press, 6th edition, 1932).

[12] L. Lichtenstein, "Uber einige Existenzprobleme der Hydrodynamik", Math. Z. 23 (1925), 89-154.

[13] L. Lichtenstein. Grundlagen der hydrodynamik (Springer, Berlin, 1929).

[14] B. McLeon, "Rearrangements", preprint (1983).

[15] D. W. Moore and P. G. Saffman, "Structure of a line vortex in an imposed strain", in Aircraft wake turbulence (eds. J. Olsen, A. Goldberg and M. Rogers), (Plenum Press, New York, 1971), 339-354.

[16] L. E. Payne, “Isoperimetric inequalities and their application”, SIAM Rev. 9 (1967), 453-458.

[17] R. T. Pierrehumbert and S. E. Widnall, "The structure of organised vortices in a free shear layer", J. Fluid Mech. 102 (1981), 301-314. 
[18] G. Polya and G. Szego, Isoperimetric inequalities in mathematical physics (Princeton Univ. Press, 1951).

[19] L. Rosenhead. "The Karman street of vortices in a channel of finite breadth", Philos. Trans. Roy. Soc. London Ser. A 228 (1929), 275-329.

[20] P. G. Saffman, "The approach of a vortex pair to a plane surface in an inviscid flow", $J$. Fluid Mech. 92 (1979), 497-503.

[21] P. G. Saffman and R. Szeto, "Structure of a linear array of uniform vortices", Stud. Appl. Math. 65 (1981), 223-248.

[22] B. Turkington, "On steady vortex !low in two dimensions, I", Comm. Partial Differential Equations 8 (1983), 999-1030.

[23] B. Turkington, "On steady vortex flow in two dimensions, II", Comm. Partial Differential Equations 8(1983), 1031-1071. 